\title{
Bioequivalence Study of Two Formulations Containing Rosuvastatin 40 Mg Tablets in Healthy Colombians
}

\author{
Vargas $\mathbf{M}^{1 *}$, Bustamante $\mathbf{C}^{2}$ and Villarraga Ea ${ }^{1}$ \\ ${ }^{1}$ Pharmacology Unit Universidad de La Sabana, Bogotá-Colombia \\ ${ }^{2}$ Universidad de La Sabana Bogotá, Colombia
}

\begin{abstract}
This is a pharmacokinetic study of two formulations containing Rosuvastatin $40 \mathrm{mg}$, with the aim to compare the Bioavailability between the Test product (Rosuvastatin made by Laboratorios Tecnoquímicas S.A., Colombia) and the Reference product (Crestor ${ }^{\circledR}$ made by Laboratorios AstraZeneca) in order to declare the Bioequivalence between both formulations. For this, an open-label, two period and two sequences previously randomized, crossover study in 30 healthy volunteers was developed, with a single $40 \mathrm{mg}$ dose in fasting conditions, a washout period of 7 days and 14 plasma samples collection between 0 and 48 hours. For the identification and assessment of plasma Rosuvastatin, an ultra-high performance liquid chromatography coupled to tandem mass spectrometer, UHPLC MS/ MS was the analytical method performed.

According to the European and FDA guidelines for Bioequivalence research, the confidence interval is within the allowed ranges for the Bioequivalence declaration and interchangeability of the Tecnoquímicas S.A. product with the Reference product.
\end{abstract}

Keywords: Bioequivalence; Rosuvastatin; Cholesterol; Statin; Pharmacokinetic

\section{Introduction}

Rosuvastatin belongs to the statin family (lovastatin, simvastatin, pravastatin, atorvastatin, etc.) and like them, acts as a competitive and selective inhibitor of the HMG-CoA reductase enzyme. It has not been demonstrated that it has the ability to avoid complications such as coronary heart disease or effects on cardiovascular morbidity and mortality [1]. From the clinical point of view, different international guidelines include the use of statins in primary and secondary cardiovascular prevention. Likewise, primary cardiovascular prevention with Rosuvastatin in normolipidemic patients with high C-reactive protein (CRP) has been demonstrated $[2,3]$.

Rosuvastatin, like other statins, has demonstrated various beneficial effects on multiple targets that could be independent of its hypolipemiant effects, but it has not been determined whether they are clinical benefits $[4,5]$. These pleiotropic effects include improvements in endothelial function [6-8], anti-inflammatory effects, cardiovascular and anti-atherosclerotic effects $[9,10]$, vascular and cardio-cerebral protective effects [11] and improvements in neural function [12].

The aim of the present study was to establish the Bioequivalence of two formulations of Rosuvastatin $40 \mathrm{mg}$ tablets, by comparing its Bioavailability after a single dose between the Test product by Tecnoquímicas S.A. (Colombia) and Crestor de AstraZeneca (Reference product).

\section{Materials and Methods}

\section{Formulations under study}

Test product: Rosuvastatin $40 \mathrm{mg}$ tablet, made and distributed by Laboratorios Tecnoquímicas S.A. in Colombia. Batch 3P3123.

Reference product: Crestor Rosuvastatin $40 \mathrm{mg}$ tablet, made and distributed by AstraZeneca. Batch KF476.

In order to declare the Pharmaceutical Equivalence between them before the development of in vivo study, physicochemical properties such as the active ingredient assessment and dosage uniformity were evaluated for the Test and Reference products and are results shown in Table 1.

Subjects: A total of 30 healthy volunteers of both genres, 15 women and 15 men, non-smokers, in a range of average age of 32 years (19 to 49 years), a mean weight of $61 \mathrm{~kg}$ ( 48 to $84 \mathrm{~kg}$ ), a mean height of $161 \mathrm{~cm}$ $(143$ to $176 \mathrm{~cm})$ and an average BMI of $23.2 \mathrm{~kg} / \mathrm{m}^{2}\left(19.0\right.$ to $\left.27.7 \mathrm{~kg} / \mathrm{m}^{2}\right)$ completed the study Table 2 .

Before the clinical phase, all volunteers went through a medical exam and laboratory tests in order to confirm their health condition. Women were also asked to perform a pregnancy test. History of alcoholism, preexisting diseases that compromised the liver or kidney

\begin{tabular}{|c|c|c|}
\hline $\begin{array}{l}\text { Physicochemical } \\
\text { characteristic }\end{array}$ & Test product & Reference product \\
\hline Description & $\begin{array}{l}\text { Round biconvex pink } \\
\text { tablet }\end{array}$ & $\begin{array}{l}\text { Oblong biconvex pink } \\
\text { tablet }\end{array}$ \\
\hline $\begin{array}{l}\text { Assessment - } \\
\text { Rosuvastatin Sodium }\end{array}$ & $105,5 \%$ of labeled & $102,7 \%$ of labeled \\
\hline Dissolution & $\begin{array}{c}94 \% \\
97 \% \\
97 \% \\
97 \% \\
102 \% \\
95 \%\end{array}$ & $\begin{array}{c}93 \% \\
94 \% \\
93 \% \\
95 \% \\
94 \% \\
101 \%\end{array}$ \\
\hline Dose uniformity & $L<15$ & $L<15$ \\
\hline
\end{tabular}

Table 1: Results of the physicochemical tests of the Test and Reference product.

*Corresponding author: Vargas M, Dentist, Pharmacologist, Scientific Director Pharmacology Unit Universidad De La Sabana, Bogotá-Colombia, Tel: 8615555 ; E-mail: mauricio.vargas@unisabana.edu.co

Received May 01, 2015; Accepted July 08, 2015; Published July 15, 2015

Citation: Vargas M, Bustamante C, Ea V (2015) Bioequivalence Study of Two Formulations Containing Rosuvastatin $40 \mathrm{Mg}$ Tablets in Healthy Colombians. J Bioequiv Availab 7: 229-232. doi:10.4172/jbb.1000245

Copyright: $\odot 2015$ Vargas M, et al. This is an open-access article distributed under the terms of the Creative Commons Attribution License, which permits unrestricted use, distribution, and reproduction in any medium, provided the original author and source are credited. 


\begin{tabular}{|c|c|}
\hline Demographic Variable & Obtained mean $(\mathbf{n}=\mathbf{3 0})$ \\
\hline Age (years) & $32 \pm 8,6$ \\
\hline Height $(\mathrm{cm})$ & $161 \pm 8,3$ \\
\hline Weight $(\mathrm{kg})$ & $61 \pm 8,8$ \\
\hline BMI $\left(\mathrm{kg} / \mathrm{m}^{2}\right)$ & $23 \pm 2,1$ \\
\hline
\end{tabular}

Table 2: Demographic data of volunteers included in the pharmacokinetic and statistical analysis.

functions, blood dyscrasia or proteinuria were considered as exclusion factors.

Medical exams and clinical laboratory tests: Complete blood count, total and direct bilirrubin, creatinine, blood glucose, total proteins, complete urine analysis, Elisa for HIV, antibodies against hepatitis $\mathrm{C}$ and $\mathrm{B}$, electrocardiogram and pregnancy test in women were asked as clinical laboratory tests.

Informed consent obtainment: The protocol and Informed Consent were authorized by the Ethic in Clinical Research Committee from the Universidad de la Sabana (CEIC), which is governed by the legal and ethical guidelines of the resolutions 008430 of 1993 and 002378 of 2008 of the Ministry of Social Protection (Colombia), of the World Conference on Harmonization for Good Clinical Practice for the institutions conducting research in human beings and the principles of the World Medical Assembly published in the Helsinki Declaration, last revision in 2013 [13].

A lecture was performed with the volunteers in order to explain the study in detail, making emphasis in the kind of drug used, dose, possible drug adverse reactions, blood volume that would be drawn in each phase of the study, the material by which these samples would be obtained, the staff on charge of obtaining them and monitoring the experience, dietary restrictions that they would have to face, and in general, all the information that they required to freely decide their enrollment in the study. Subsequently, each one of them signed an informed consent form.

Study design: An open-label, randomized, two period, two sequences, crossover with a washout period of 7 days design was used. Three days before the beginning of each period, volunteers had to abstain from taking any kind of drugs, alcohol or any food or drink that contained methylxanthines. These restrictions were maintained during all the sampling time. All volunteers were randomly assigned to a treatment sequence.

Drug administration: Volunteers had a 10 hours fasting previous to the drug administration, which was delivered with $200 \mathrm{~mL}$ of water in a $40 \mathrm{mg}$ dose of Rosuvastatin [5], that means 1 tablet of $40 \mathrm{mg}$ to each volunteer and two hours later, standardized feed was given to each one of the volunteers. Three complete meals (breakfast, lunch and dinner) and two snacks (one in the morning and one in the afternoon) were given during the hospitalization period.

The sampling team consisted on a physician and a licensed nurse. Immediately before administering the drug, a blood sample was obtained by venipuncture in the arm using a Vacutainer, which was called the time zero sample. All volunteers received the Test product or Reference product according to the randomization, and 14 venous blood samples were taken according to the following times: $0 ; 2,3,3,5$, $4,4.5,5,6,7,8,10,24,36$ and 48 hours. Samples were labeled for their identification and centrifuged at $3000 \mathrm{rpm}$ for 30 minutes. Plasma was transferred into a previously labeled tube and frozen at $-20^{\circ} \mathrm{C}$ for later analysis. Administration was repeated after 7 days of washing time, completing the second study period.
Analytical method validation: The validation was performed in compliance with the validation procedure of bioanalytical methodology established by QUASFAR M\&F S.A. (PL-021) which meets the FDA suggestions to demonstrate a suitable specificity, linearity, precision, and accuracy [13].The bioanalytical method employed for Rosuvastatin quantification in plasma was ultra-high-performance liquid chromatography-tandem mass spectrometry (UHPLC-MS/MS) in electrospray ionization (ESI) mode [14-16].

Plasma samples were acidulated with methanol and acetic acid to form an ionic complex with tetra-n-butylammoniumhydroxide (0.5\%). Liquid-liquid extraction was made with ethyl acetate. Analyte separation was achieved with a Zorbax Eclipse Plus C18 RHHD $2.1 \mathrm{x}$ $50 \mathrm{~mm}, 1.8 \mu \mathrm{m}$ Column. Isocratic elution was performed with a mobile phase comprised by Buffer:MeOH 20:80 (Buffer: Formic acid 2\%). Total run time was $1.5 \mathrm{~min}$. Rosuvastatin was detected with an ESI+ ionizaton mode, and monitoring the 482.2 --- $258.2 \mathrm{~m} / \mathrm{z}$ transition. UHPLC injection volume was $10.0 \mu \mathrm{L}$.

Pharmacokinetic analysis: The pharmacokinetic analysis was performed using the WinNonlin 5.3 software (Pharsight Corporation, Cary USA), by a non-compartmental analysis. The maximum concentration $\left(\mathrm{C}_{\max }\right)$ and time to reach it $\left(\mathrm{t}_{\max }\right)$ were directly obtained from the plasma concentration results, as the FDA [17] and the European Medicines Agency (EMA) [18] recommend. The $\mathrm{AUC}_{\text {total }}$ was calculated by the sum of partial AUC: a) $\mathrm{AUC}_{0-\mathrm{f}}$, between time zero and the last time with detectable concentrations, calculated by the trapezoidal rule, and guaranteeing the calculation of at least the $80 \%$ AUC with the last sample, b) $\mathrm{AUC}_{\mathrm{t}-\infty}$ calculated as the $\mathrm{C} / \mathrm{K}$ ratio, being $\mathrm{C}$ the last detectable concentration and $\mathrm{K}$ the slope of the curve obtained by linear regression of the points corresponding to the elimination phase of the drug by linear regression of the natural logarithm of the concentrations [10]. The elimination rate constant $(\mathrm{Ke})$, half-life $\left(\mathrm{t}_{1 / 2}\right)$, clearance $(\mathrm{Cl})$ and the mean residence time (MRT), adjusted to Bioavailability, were calculated after performing the non-compartmental analysis. The pharmacokinetic variables results are summarized in Table 3, with the $\mathrm{C}_{\max }, \mathrm{AUC}_{0-\mathrm{t}}, \mathrm{AUC}_{0-\infty}, \mathrm{T}_{\max }$ and elimination rate constant (Ke) values of each of the studied formulations.

Statistical analysis: Analysis of variance (ANOVA) was used to determine possible effects for each variation factor for sequence, period or subjects. For this, the F-test was used with a $5 \%(\alpha=0.05 \%)$ statistical significance. Statistical comparison of the transformed pharmacokinetic parameters of both formulations was made using the WinNonlin version 5.3 statistical programs. The following Bioequivalence criteria was established in the protocol: the $90 \%$ confidence interval of Test $\mathrm{C}_{\max }$ / Reference $\mathrm{C}_{\max }$ and Test last AUC / Reference last AUC, ratios that must be within the $80-125 \%$ acceptability range. Besides, the last parameter AUC must not be less than the $80 \%$ of the total AUC parameter.

Adverse events report: There were no adverse events, which was recorded according to the INVIMA (Colombian regulatory authority) guidelines, Provision No (1067/08), which defines the event as severe or not severe, and then in function of its definition, as probably, possible or not related with the drug study. Cases are only reported as received from the research unity and without statistical estimation, since the sample size does not have sufficient statistical power.

\section{Results}

Analytical results of the active ingredient content, dose uniformity and dissolution test complied with the specifications required for the 
declaration of Pharmaceutical Equivalence, as is shown in Table 1. The comparative dissolutions profiles show a difference factor $\left(f_{1}\right)$ of 2,6 , within the $0-15$ compliance range and a similarity factor $\left(f_{2}\right)$ of 74 that equally accomplishes with the compliance range 50-100 for this variable.

The analytical method showed to be specific, since there was no interference between the components of the matrix in the identification and quantification of Rosuvastatin. Precision showed coefficients of variation below $15 \%$ for low (1.0 ng/mL, CV: 8,33\%), medium (50 ng/ $\mathrm{mL}, \mathrm{CV}: 4,36 \%)$ and high (100 ng/mL, CV: 2,21\%) levels. Accuracy was determined comparing the relation of the sample areas against 5 calibration curves of the system and deviations below $20 \%$ were obtained for the lower concentrations of the curve and below $15 \%$ for the other concentrations, thus complying with the specification. The quantification limit was $1 \mathrm{ng} / \mathrm{mL}$ and the detection limit was $0.1 \mathrm{ng} / \mathrm{mL}$.

A total of 30 healthy Colombian volunteers of both genres $(50 \%$ women and $50 \%$ men) participated in the present study, completing both periods and were included in the pharmacokinetic and statistical analysis. Demographic data are summarized in Table 2. Both treatments were well tolerated, without the presence of adverse events.

Table 3 shows the mean pharmacokinetic parameters obtained from all volunteers (mean \pm SD) and Table 4 shows the $90 \%$ confidence intervals of logarithmically transformed pharmacokinetic parameters, analysis performed in order to determine Bioequivalence between the Test product by Tecnoquímicas S.A. and the Reference by Crestor AstraZeneca.

\section{Discussion}

Controlling drug costs is a constant search from governments [19], therefore the use of multisource products that demonstrate safety and good risk / benefit profile, through validated research to do so, such as Bioequivalence studies, that allow the interchangeability between generic vs. Reference products without the need of repeating the clinical studies in patients [20] is the best option for patients and the financial system of a country.

WHO recommends in its guidelines for conduction of studies of comparative Bioavailability, to perform in vivo test on the multisource products in order to evaluate a dose and a sudden increase of plasma drug concentration, which was evaluated in the present study [20].

\begin{tabular}{|c|c|c|}
\hline Parameter (unit) & Test product & Reference product \\
\hline $\mathbf{C}_{\max }$ (ng/mL) & $21,1 \pm 9,6$ & $20,4 \pm 10,2$ \\
\hline AUC $_{\mathbf{0 - t}}$ (ng.h/mL) & $191,7 \pm 94,2$ & $194,1 \pm 97,0$ \\
\hline AUC $_{\mathbf{0 - \infty}}$ (ng.h/mL) & $234,6 \pm 126,8$ & $298,0 \pm 176,4$ \\
\hline $\mathbf{T}_{\max }$ (h) & $3,2 \pm 1,2$ & $3.3 \pm 1,1$ \\
\hline $\mathbf{K e}(\mathbf{1} / \mathbf{h})$ & $0,113 \pm 0,166$ & $0,082 \pm 0,103$ \\
\hline
\end{tabular}

Table 3: Pharmacokinetic parameters of Rosuvastatin of Test product (Tecnoquímicas S.A.) and Reference product (Crestor $\left.{ }^{\circledR}\right)$ followed by a single ora dose of $40 \mathrm{mg}$ on fasting state.

\begin{tabular}{|c|c|c|c|c|} 
& Units & Ratio\% & \multicolumn{2}{|c|}{$\begin{array}{c}\mathbf{9 0 \%} \text { standard Cl (Test/ } \\
\text { Reference) }\end{array}$} \\
\hline Ln(Tmax) & $\mathrm{hr}$ & 95,9 & 83,5 & 110,2 \\
\hline Ln(Cmax) & $\mathrm{ng} / \mathrm{mL}$ & $\mathbf{1 0 4 , 2}$ & $\mathbf{8 9 , 5}$ & $\mathbf{1 2 1 , 4}$ \\
\hline Ln(AUClast) & $\mathrm{hr} r^{*} \mathrm{ng} / \mathrm{mL}$ & $\mathbf{9 6 , 9}$ & $\mathbf{8 2 , 0}$ & $\mathbf{1 1 4 , 6}$ \\
\hline Ln(AUCall) & $\mathrm{hr}$ (A) $\mathrm{ng} / \mathrm{mL}$ & 98,0 & 84,3 & 113,9 \\
\hline
\end{tabular}

Table 4: $90 \%$ confidence intervals of logarithmic transformed pharmacokinetic parameters of two formulations containing Rosuvastatin (Test and Reference product) following administration to healthy volunteers.

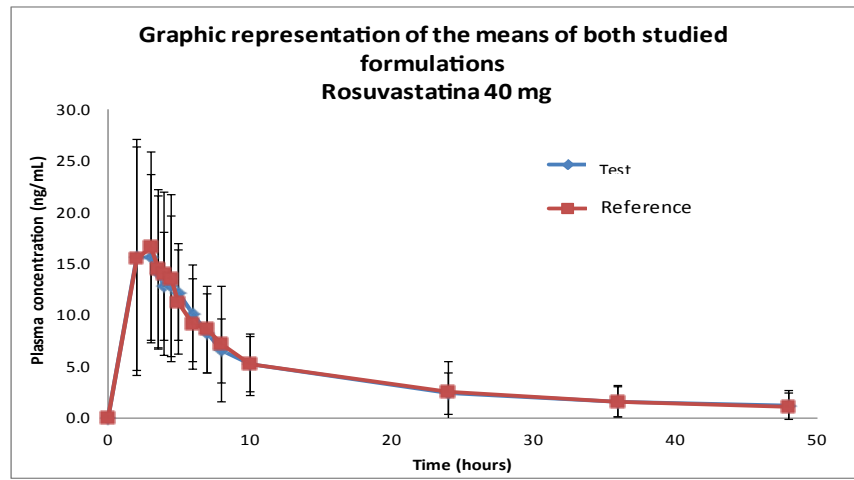

Figure 1: Bioavailability curve (Concentration vs. Time) obtained following a dose on fasting state of Rosuvastatin $40 \mathrm{mg}$ of the Test product (Tecnoquímicas S.A.) and the Reference product (Crestor ${ }^{\circledR}$ de AstraZeneca)

The statement of Pharmaceutical Equivalence allowed qualifying the in vitro quality attributes of both formulations. The design of this study with healthy volunteers, two periods, two sequences, single dose, crossover, minimizes variability and allows evaluating the effects of the formulation. The analytical method used was selective, precise, accurate and robust. All the 30 volunteers completed the study and did not present adverse events with neither of the studied formulations. The washout period was longer than the recommended 7 elimination halflives and guaranteed the absence of carryover effect between periods.

Li et al. [21] and Gao et al. [22] reported similar pharmacokinetic values for $\mathrm{C}_{\max }, \mathrm{T}_{\max }$ and AUC in studies carried out in Chinese healthy volunteers to those reported in our study with healthy Colombian volunteers. There were no gender differences either. Adverse reactions to Rosuvastatin were minimal in both populations.

However, they also evaluated pharmacokinetics in fed conditions and they reported lower $\mathrm{C}_{\max }$ and AUC values when administered in fed conditions compared to fasting conditions, which suggests that Rosuvastatin should be administered with an empty stomach to avoid an absorption decrease.

The aim of the present study was to evaluate the Bioequivalence between two formulations of Rosuvastatin $40 \mathrm{mg}$. Figure 1 shows the curves of mean plasma concentration vs. time graphics, where similarity can be observed. Moreover, the means of $\mathrm{AUC}_{0-\mathrm{t}}$ and $\mathrm{C}_{\max }$ were not significantly different and the $90 \%$ confidence intervals of ratio (Test/ Reference) for the logarithmically transformed mean criteria of $\mathrm{AUC}_{0-\mathrm{t}}$ and $\mathrm{C}_{\text {max }}$ comply with the range established by the FDA [17] and EMA [18] (Table 4).

For Rosuvastatin, incorporating a Bioequivalent form to pharmacological treatment of dyslipidemia contributes with the clinical objective of primary and secondary prevention of cardiovascular disease.

In response to this evidence, the present study performed between the Test product by Tecnoquímicas S.A. and Crestor by AstraZeneca in Colombian population, shows the possibility of interchangeability between these two formulations. [17-19,23].

\section{Conclusion}

Rosuvastatin formulation made by Tecnoquímicas S.A., Test product, and the formulation made by AstraZeneca (Crestor'), Reference product, have pharmacokinetic parameters that allow declaring Bioequivalence between both formulations. 
Citation: Vargas M, Bustamante C, Ea V (2015) Bioequivalence Study of Two Formulations Containing Rosuvastatin 40 Mg Tablets in Healthy Colombians. J Bioequiv Availab 7: 229-232. doi:10.4172/jbb.1000245

*The test product is manufactured by Laboratorios Tecnoquímicas S.A. in Jamundí, Colombia and commercialized in Colombia and Ecuador as Rosuvastatina $\mathrm{MK}^{*}$ and in Central America as Rosuvastateg $\mathrm{TG}^{*}$, the reference product is manufactured by Laboratorios IPR Pharmaceuticals Inc., in Puerto Rico, and commercialized in Colombia by AstraZeneca Colombia S.A. as Crestor.

\section{References}

1. Martin PD, Warwick MJ, Dane AL, Hill SJ, Giles PB (2003) Metabolism, excretion, and pharmacokinetics of Rosuvastatin in healthy adult male volunteers. Clin Ther 25: 2822-2835.

2. Nccpc The National Collaborating Center For Primary Care (2010) Guideline Lipid Modification: Cardiovascular risk assessment and the modification of blood lipids for the primary and secondary prevention of cardiovascular disease. Royal College of General Practitioners.

3. Ridker PM, Danielson E, Fonseca FA, Genest J, Gotto AM Jr, et al. (2008) Rosuvastatin to prevent vascular event in men and women with elevated C-reactive protein. New Engl J Med 359: 2195-2207.

4. LaRosa JC (2001) Pleiotropic effects of statins and their clinical significance. Am J Cardiol 88: 291-293.

5. Bonetti PO, Lerman LO, Napoli C, Lerman A (2003) Statin effects beyond lipid lowering--are they clinically relevant? Eur Heart J 24: 225-248.

6. Laufs U, Bohm M, Gertz K (2002) The hydrophilic HMG-CoA reductase inhibitor Rosuvastatin regulates endothelial NO synthase and ischaemic stroke in vivo. Circulation 106: II272

7. Hermann M, Ruschitzka F, Camici G, Luescher TF (2002) Rosuvastatin improves endothelial dysfunction in a rat model of normocholesterolemic mineralocorticoid hypertension. Hypertension 40: 439-440.

8. Stalker TJ, Lefer AM, Scalia R (2001) A new HMG-CoA reductase inhibitor, rosuvastatin, exerts anti-inflammatory effects on the microvascular endothelium: the role of mevalonic acid. Br J Pharmacol 133: 406-412.

9. Werner N, Priller J, Laufs U, Endres M, Böhm M, et al. (2002) Bone marrowderived progenitor cells modulate vascular reendothelialization and neointimal formation: effect of 3-hydrox-y-3-methylglutaryl coenzyme a reductase inhibition. Arterioscler Thromb Vasc Biol 22: 1567-1572.

10. Susic D, Varagic J, Ahn J, Slama M, Frohlich ED (2003) Beneficial pleiotropic vascular effects of rosuvastatin in two hypertensive models. J Am Coll Cardiol 42: 1091-1097.

11. Jones SP, Gibson MF, Rimmer DM 3rd, Gibson TM, Sharp BR, et al. (2002)
Direct vascular and cardioprotective effects of rosuvastatin, a new HMG-CoA reductase inhibitor. J Am Coll Cardiol 40: 1172-1178.

12. Nangle MR, Cotter MA, Cameron NE (2003) Effects of rosuvastatin on nitric oxide-dependent function in aorta and corpus cavernosum of diabetic mice: relationship to cholesterol biosynthesis pathway inhibition and lipid lowering. Diabetes 52: 2396-2402.

13. Declaración de Helsinki de la Asociación médica Mundial (2013). Principios éticos para las investigaciones médicas en seres humanos $64^{\text {a }}$ Asamblea General, Fortaleza, Brasil.

14. Sharpless KE, Thomas JB, Duewer DL, Putzbach K, Rimmer CA, et al. (2007) Preparation and characterization of standard reference material 3276, carrot extract in oil. Anal Bioanal Chem 389: 207-217.

15. Tiwari G, Tiwari R (2010) Bioanalytical method validation: An updated review. Pharm Methods 1: 25-38.

16. Wille SMR, Peters FT, Di Fazio V, Samyn N (2011) Practical Aspects concerning Validation and Quality Control for Forensic and Clinical Bioanalytical Quantitative Methods. Accred Qual Assur 16: 279-292.

17. Chow SC, Shao J, Hu OY (2002) Assessing sensitivity and similarity in bridging studies. J Biopharm Stat 12: 385-400.

18. The European Agency for the Evaluation of Medicinal Products, EMEA (2001) Committee for Proprietary Medicinal Products (CPMP). Note for guidance on the investigation of Bioavailability and Bioequivalence, London.

19. Perry R (2010) Perspectives on the bioequivalence and therapeutic equivalence of generic formulations: An overview of the landscape. Clin Ther 32: 17961797.

20. WHO Expert Committee on Specifications for Pharmaceutical Preparations (2006) WHO expert committee on specifications for pharmaceutical preparations. Fortieth report. World Health Organ Tech Rep Ser 937: 1-461.

21. Li Y, Jiang X, Lan K, Zhang R, Li X, et al. (2007) Pharmacokinetic Properties of Rosuvastatin After Single-Dose, Oral Administration in Chinese Volunteers: A Randomized, Open- Label, Three-Way Crossover Study. Clin Ther 29: 2194 2203

22. Gao J, Zhong D, Duan X, Chen X (2007) Liquid chromatography/negative ion electrospray tandem mass spectrometry method for the quantification of rosuvastatin in human plasma: application to a pharmacokinetic study. $J$ Chromatogr B Analyt Technol Biomed Life Sci 856: 35-40.

23. Multisource (Generic) Pharmaceuticals Products: Guidelines On Registration Requirements to Establish Interchangeability, WHO Expert Committee On Specifications for Pharmaceutical Preparations, Thirty Four Report (WHO Technical Report Series, N" 9337), WHO 22006. 